\title{
Monte Carlo simulation of a single ring among linear chains: Structural and dynamic heterogeneity
}

\author{
Yong-Biao Yang, ${ }^{1}$ Zhao-Yan Sun, ${ }^{1}$ Cui-Liu Fu, ${ }^{1}$ Li-Jia An, ${ }^{1, a), b)}$ and Zhen-Gang Wang ${ }^{2, a), c)}$ \\ ${ }^{1}$ State Key Laboratory of Polymer Physics and Chemistry, Changchun Institute of Applied Chemistry, \\ Chinese Academy of Sciences, Changchun 130022, People's Republic of China \\ ${ }^{2}$ Division of Chemistry and Chemical Engineering, California Institute of Technology, \\ Pasadena, California 91125, USA
}

(Received 23 November 2009; accepted 30 June 2010; published online 10 August 2010)

\begin{abstract}
We perform lattice Monte Carlo simulation using the bond-fluctuation model to examine the conformation and dynamic properties of a single small flexible ring polymer in the matrix of linear chains as functions of the degree of polymerization of the linear chains. The average conformation properties as gauged by the mean-square radius of gyration and asphericity parameter are insensitive to the chain length for all the chain lengths examined $(30,100,300$, and 1000). However, in the longer chain (300 and 1000) samples, there is an increased spread in the distribution of the value of these quantities, suggesting structural heterogeneity. The center-of-mass diffusion of the ring shows a rapid decrease with increasing chain length followed by a more gradual change for the two longer chain systems. In these longer chain systems, a wide spread in the value of the apparent self-diffusion coefficient is also observed, as well as qualitatively different square displacement trajectories among the different samples, suggesting heterogeneity in the dynamics. A primitive path analysis reveals that in these long chain systems, the ring can exist in topologically distinct states with respect to threading by the linear chains. Threading by the linear chain can dramatically slow down and in some cases stall the diffusive motion of the ring. We argue that the life times for these topological conformers can be longer than the disentanglement time of the linear chain matrix, so that the ring exhibits nonergodic behavior on time scales less or comparable to the life time of these conformers. Our results suggest a picture of the ring diffusion as one where the diffusion path consists of distinctive segments, each corresponding to a different conformer, with slow interconversion between the different conformers. (c) 2010 American Institute of Physics.
\end{abstract}

[doi:10.1063/1.3466921]

\section{INTRODUCTION}

The conformation and dynamics of nonconcatenated ring polymers have attracted much attention during the last few decades because of their unique topology. Since rings have no ends, they cannot "reptate" like linear chains, or "retract" like branched polymers. The diffusion mechanism of rings has been suggested theoretically as amoebalike motion: they thrust and pull on unentangled loops. ${ }^{1,2}$ For ring polymers in bulk, in spite of extensive work using experiments, ${ }^{3-10}$ theories, ${ }^{11-14}$ and simulations, ${ }^{15-23}$ many controversies still remain.

Flory's scaling exponent for the radius of gyration $R_{\mathrm{g}}^{2}$ $\sim N_{\text {Ring }}^{2 v}$, for example, has not been agreed upon among different studies. Using a self-consistent argument, Cates and Deutsch ${ }^{11}$ predicted the value of $v$ for ring polymers in melt to be 0.4 at $N_{\text {Ring }} \rightarrow \infty$. This result is supported by the simulation work of Müller et al. ${ }^{15}$ using the bond fluctuation model (BFM). On the other hand, Brown and Szamel, ${ }^{16,17}$ also using the BFM, found the value of $v$ to be 0.42 in the range of $100 \leq N_{\text {Ring }}<500$. With the same BFM, Suzuki

\footnotetext{
${ }^{a}$ Authors to whom correspondence should be addressed.

${ }^{b}$ Electronic mail: 1jan@ @iac.j1.cn.

c)Electronic mail: zgw@caltech.edu.
}

et $a .^{23}$ simulated ring polymers with $N_{\text {Ring }}$ as large as 1000 and concluded that $v$ is bracketed by $1 / 3 \leq v<0.365$. Experimentally, at least two different values of $v$ for ring polymers in melt have been reported: 0.42 by Arrighi et al. ${ }^{8}$ and 0.34 by Takano,${ }^{10}$ both from neutron scattering data.

Similar disagreement exists concerning the critical entanglement chain length $\left(N_{\mathrm{c}}\right)$ for rings in the melt. Some viscosity studies suggested that $N_{\mathrm{c}}$ for rings is smaller than that of linear polymers, ${ }^{3,4,6}$ but simulation results argued that $N_{\mathrm{c}}$ for rings would be about two to five times higher than that of linear chains, ${ }^{15}$ or even much larger. ${ }^{17}$ Moreover, by demonstrating the self-similar dynamics of purified high-molarmass ring polymers, Kapnistos et al. ${ }^{24}$ even questioned the existence of $N_{\mathrm{c}}$ for ring polymers.

For both conformation and dynamic properties of ring polymers in bulk, the differences are notable and these differences may be due to various causes. Factors such as study methods and the range of $N_{\text {Ring }}$ studied are certainly worth considering, but the purity of samples may be the most important one because most synthetic rings in experiments are in fact mixtures of rings and linear chains due to contamination or limitation in the purification methods. Thus it is of both fundamental and practical importance to study the properties of ring polymers in ring-linear blends.

There have been several recent studies focused on the 


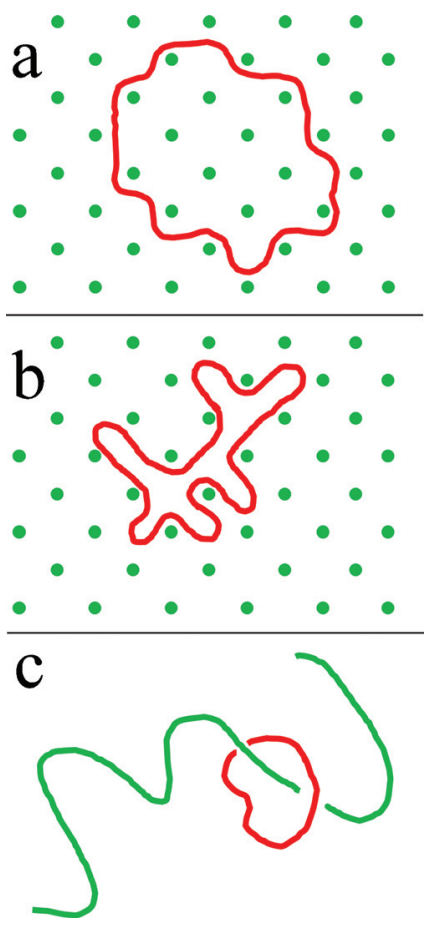

FIG. 1. Schematic configurations for a ring polymer in a linear chain matrix envisioned by the three mechanisms proposed in the literature (linear chains are represented by green points or green line): (a) the ring can be pinned or threaded so that it only moves by constraint release (CR); (b) the ring is not pinned but assumes a ramified conformation with loops and moves by reptation (similar to a linear molecule of half of its length) (RR); (c) the ring is threaded by a single chain and moves along the chain contour (R1).

properties of ring polymers in the ring-linear blends. ${ }^{24-31}$ Iyer et $a{ }^{28}{ }^{28}$ proposed a scaling model on the size of ring polymers in ring-linear blend and found that by gradually increasing the composition of linear chains, the ring molecules swell, with Flory's scaling exponent $v$ increasing from 0.4 to 0.5 in the limit of infinite dilution for the rings. Using the BFM, Subramanian and Shanbhag ${ }^{29,30}$ studied the dynamics of entangled ring polymers in ring-linear blends and found the self-diffusion coefficient and the primitive path length of ring polymers are more sensitive to volume fraction than that of linear chains. This is in agreement with the results of Robertson et al. on DNA..$^{25-27}$ By tracking the Brownian motion of individual tracer DNA molecules in blend of four topological combinations, Robertson et al. found that the molecular topology, especially for the case of circular DNA molecules surrounded by linear molecules, have a strong effect on the diffusion at above certain concentration or molecular weight. Their findings suggest that free ends play a critical role in generating entanglements which retard diffusion. They argued that the degree of penetration or threading by the linear chains is the key factor to influence DNA diffusion.

Earlier experiments examined the matrix and topology effects in ring-linear blends and several mechanisms have been put forward. If large rings are threaded by many linear chains [Fig. 1(a)], reptation is thought to be impossible and the diffusion of the rings are supposed to occur via a constraint release $(\mathrm{CR})$ mechanism, which was originally proposed to describe a long enough linear chain diffusing in the matrix of short chains; the CR mechanism predicts $D_{\mathrm{CR}}$ $\sim N_{\text {Ring }}^{-1} N_{\text {Linear }}^{-3}$ (Ref. 32) for the self-diffusion coefficient. In the opposite limit $N_{\text {Ring }} \ll N_{\text {Linear }}$, Klein ${ }^{33}$ proposed an alternative mechanism, the restricted reptation (RR) mechanism [Fig. 1(b)]. He argued that rings in fixed obstacle lattice will assume conformations like amoebae and form double-folded linear conformations and diffuse as reptation, in which $D$ should obey $D_{\text {RR }} \sim N_{\text {Ring }}^{-2} \exp \left(-\beta N_{\text {Ring }}\right)$. The CR and RR thus describe two opposite limits: in the former, the rings are relatively large and are threaded by many linear chains, while in the latter, the rings are relatively small with no threading by the linear chains. Another mechanism referred to as once-threaded model (R1), i.e., Fig. 1(c), was proposed by Mills et al., ${ }^{34}$ which predicts $D_{\mathrm{R} 1} \sim N_{\text {Ring }}^{-1} N_{\text {Linear }}^{-1}$. In the R1 picture, the ring diffuses along, like a smoke ring, constrained to follow the contour of the linear chains. These three mechanisms are mainly distinguished by the degree of threading; the greater the degree of threading, the more strongly the diffusion coefficient decreases with the molecular weight of matrix linear chains (the scaling exponent for $N_{\text {Linear }}$ changes from -3 to -1 to 0 when diffusion mechanism changes from $\mathrm{CR}$ to $\mathrm{R} 1$ to RR). However, it is speculated that the diffusion process of a single ring in linear matrix is a combination of them. ${ }^{34}$

Considering the different ideas (CR, RR, and R1) about the dynamics of ring polymers in the matrix of linear chains, the motivation of the present study is to understand the individual behavior of ring polymers in the "sea" of matrix of linear chains at the molecular level. Using Monte Carlo method, we study the conformation and dynamics of a single ring of relatively small size (ring-30) in the linear matrix with several different chain lengths (linear-30, 100, 300, 1000). The choice of a single ring in our system allows us to more thoroughly examine the (topological) interaction between the ring and the linear matrix chains without the additional complications due to ring-ring interactions. We choose the chain length of the ring to be 30 , so that it is large enough to be flexible and relatively free of lattice artifact, but not too large as to allow multiple entanglements between the ring and a single chain (e.g., multiple winding of the chain by the ring or vice versa).

We find that, on time scales up to the relaxation time of the matrix chains, heterogeneity is observed for both the static and dynamic properties of the ring. At a given length of the linear chains, a ring may be threaded once, twice, thrice, etc., or not at all. For very long chains, interconversion between the different threading states becomes difficult, which is responsible for the observed heterogeneity. Thus for long matrix linear chains, rings with different threading states have different individual behavior in a substantial span of time scales, much reminiscent of the "molecular individualism" observed in the multiple conformational states of long DNA molecules in shear flows. ${ }^{35,36}$ From a different perspective, when the size of the linear chains is much larger than that of the ring, the ring has to diffuse in an environment whose local isotropy of space is broken. This results in strong correlation in the ring's motion.

The rest of the paper is organized as follows. The next section briefly describes the model and simulation protocols. 
TABLE I. Parameters and some results of the simulation: degree of polymerization of ring polymer $\left(N_{\text {Ring }}\right)$ and linear polymer $\left(N_{\text {Linear }}\right)$; length of simulation box, $L_{\text {box }}$; equilibration time, $\tau_{\mathrm{eq}}$; simulation time, $\tau_{\text {run }}$; radius of gyration, $\left\langle R_{\mathrm{g}}^{2}\right\rangle$; asphericity parameter, $\langle A\rangle$; and the center-of-mass self-diffusion coefficient, $D_{\mathrm{cm}}$. Times are measured in MCS and distances in units of the lattice constant. Note that for the data of ring-30 melt, their error bars are negligible.

\begin{tabular}{cccccccc}
\hline \hline$N_{\text {Ring }}$ & $N_{\text {Linear }}$ & $L_{\text {box }}$ & $\left(\times 10^{-5}\right)$ & $\begin{array}{c}\tau_{\text {run }} \\
\left(\times 10^{-5}\right)\end{array}$ & $\left\langle R_{\mathrm{g}}^{2}\right\rangle$ & $\langle A\rangle$ & $\begin{array}{c}D_{\text {cm }} \\
\left(\times 10^{4}\right)\end{array}$ \\
\hline 30 & $($ Melt $)$ & 20 & 1.0 & 5.0 & 6.12 & 0.2351 & 3.20 \\
30 & 30 & 20 & 1.0 & 1000 & $6.68 \pm 0.0$ & $0.2394 \pm 1 \times 10^{-4}$ & $2.30 \pm 0.03$ \\
30 & 100 & 30 & 100 & 1000 & $6.68 \pm 0.01$ & $0.2391 \pm 1 \times 10^{-4}$ & $1.35 \pm 0.02$ \\
30 & 300 & 30 & 100 & 1000 & $6.71 \pm 0.05$ & $0.2393 \pm 4 \times 10^{-4}$ & $1.01 \pm 0.05$ \\
30 & 1000 & 40 & 2000 & 5000 & $6.63 \pm 0.08$ & $0.2383 \pm 6 \times 10^{-4}$ & $0.911 \pm 0.08$ \\
\hline \hline
\end{tabular}

Results are presented and discussed in Sec. III, where we show data for several conformation and dynamic properties. We find that while the average size and shape of a ring remain essentially the same for the different matrix chain lengths, their distribution from sample to sample becomes much wider in the case of long linear chains, reflecting the slowing down of interconversion between different conformation states. The average self-diffusion coefficient decreases with increasing length of the matrix chains and with accompanying increase in the spread of the data, reflecting the dynamic heterogeneity. We examine this dynamic heterogeneity by tracking the trajectory of individual rings in different samples and by an analysis of the non-Gaussian parameter. A primitive-path analysis is used to provide direct visualization of the different threading states of the ring by the chains. In Sec. IV, we summarize our main results and offer some concluding remarks.

\section{MODEL AND SIMULATION METHODS}

In this simulation we use Shaffer's bond fluctuation model (S-BFM). ${ }^{37,38} \mathrm{~S}-\mathrm{BFM}$ has been used to model bulk polymer melts and extended to the blends of ring and linear polymers. ${ }^{28-30}$ This model has two remarkable advantages. One is that the number of chain segments between entanglements $\left(N_{\mathrm{e}}\right)$ (Ref. 39) for linear chains in melt, is quite small $\left(N_{\mathrm{e}} \approx 30\right),{ }^{37,38,40}$ which makes it easy to simulate entangled linear chains with a relatively large $N_{\text {Linear }} / N_{\mathrm{e}}$. The other advantage is that the chain crossing can be simply switched "on" and "off," which can be used to control the entanglement between chains without affecting the static properties or the local segment mobility. All this makes it possible to obtain equilibrium conformation for noncrossing chains (or real chains) within a relatively short period by starting from equilibrated crossing chains. ${ }^{37}$

In our simulations, monomers are placed on a $3 \mathrm{D}$ cubic lattice with size $L_{\mathrm{box}} \times L_{\mathrm{box}} \times L_{\mathrm{box}}$ (for details see Table I). Admittedly, our box size for the linear-1000 system (40 $\times 40 \times 40$ ) is not very large, and as a result, the system may suffer from some "overlap" artifacts due to the periodic boundary conditions. The main effect of such artifacts will be on the quantitative dependence of the ring properties on the linear-chain length, which is not our focus here. Our emphasis is on the qualitative behavior of an isolated ring-30 in the presence of long chains, which we believe to be unaffected by such artifacts. The total fractional occupancy of the lattice is fixed at $\varphi=\varphi_{\text {Ring }}+\varphi_{\text {Linear }} \approx 0.5$, which has been shown to be sufficiently high to describe meltlike systems. ${ }^{41}$ Monte Carlo step (MCS) is defined as a trial event for attempting moving every monomer in the box once. Throughout this article, chain length is expressed in number of monomers and time in MCS. The studied model systems and some obtained properties are listed in Table I (the data of ring melt are included for comparison). For each model system, 50 independent runs are performed. For those systems, the ratios $N_{\text {Linear }} / N_{\mathrm{e}}$ are about 1.0, 3.3, 10.0, and 33.3 for linear-30, 100, 300, and 1000 samples, respectively. For simplicity of notation, these systems will be henceforth denoted by R30/ L30, R30/L100, R30/L300, and R30/L1000, respectively.

We obtain the equilibrium configurations using the following steps. First, the initial configurations are generated by putting the linear chains on the lattice as ordered thin flakes and the single ring as a compact loop. Then $\tau_{\text {eq }}$ MCS are run to equilibrate the system. In this step, chain crossing for linear-linear and linear-ring is allowed; as for the ring itself, however, chain crossing is always forbidden to avoid concatenated or knotted rings. $\tau_{\text {eq }}$ is determined using a similar method as Shaffer's, ${ }^{37}$ i.e., $\tau_{\text {eq }}$ is at least ten times as big as the longest relaxation time of the crossing linear chains in melt. After that we run the simulation for more than $\tau_{\text {eq }} / 10$ to gradually remove those chain crossing. Then the equilibrium configurations are obtained and simulations are run $\tau_{\text {run }}$ MCS to collect data $\left(\tau_{\text {run }}\right.$ is listed in Table I).

\section{RESULTS AND DISCUSSION}

Before studying the diffusion behavior of a ring polymer in the linear chain matrix, we first examine the equilibrium ring conformation, which we characterize by the mean square radius of gyration $\left\langle R_{\mathrm{g}}^{2}\right\rangle$ and the asphericity parameter $\langle A\rangle{ }^{42}$ defined, respectively, as

$$
\left\langle R_{\mathrm{g}}^{2}\right\rangle=\left\langle\lambda_{1}+\lambda_{2}+\lambda_{3}\right\rangle
$$

and

$$
\langle A\rangle=\left\langle\frac{\sum_{i>j}^{3}\left(\lambda_{i}-\lambda_{j}\right)^{2}}{2\left(\sum_{i=1}^{3} \lambda_{i}\right)^{2}}\right\rangle,
$$

where $\lambda_{1}, \lambda_{2}$, and $\lambda_{3}$ are the squares of three principal radii of gyration of the polymer. As defined in Eq. (2), $\langle A\rangle$ is a number between 0 and 1: $\langle A\rangle=0$ corresponds to a perfect 


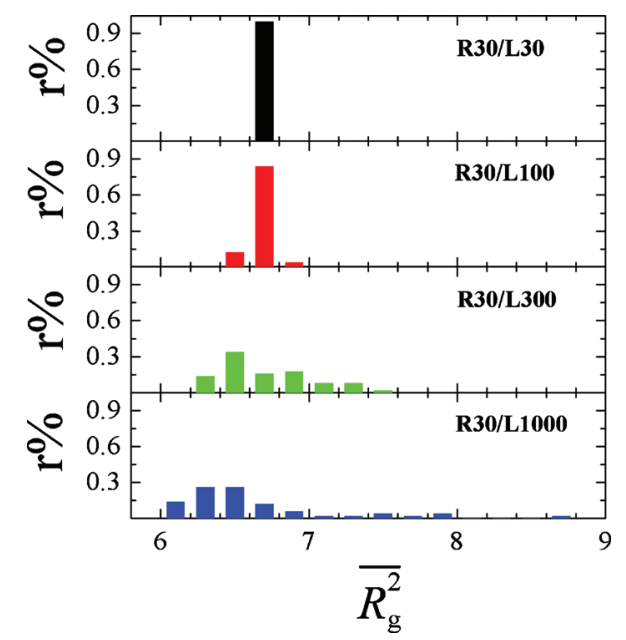

FIG. 2. Percentage distribution of the mean square radius of gyration from 50 samples for different chain lengths of the matrix.

sphere, and the deviation of $\langle A\rangle$ from 0 indicates the extent of anisotropy.

The simulated $\left\langle R_{\mathrm{g}}^{2}\right\rangle$ and $\langle A\rangle$ of ring-30 in its own melt and in linear matrix are listed in Table I. We see that a ring in linear matrix is more expanded and its shape deviates from a perfect sphere more than that in melts. For the long linear chain systems, $\left\langle R_{\mathrm{g}}^{2}\right\rangle$ and $\langle A\rangle$ for the ring are nearly independent of $N_{\text {Linear }}\left(\left\langle R_{\mathrm{g}}^{2}\right\rangle\right.$ is about 6.67 and $\langle A\rangle$ is about 0.2389$)$. The expansion of the ring by linear chains is to be expected and is consistent with earlier work on ring-linear blend. Iyer et $a{ }^{28}{ }^{28}$ suggested that the size of ring increases as its concentration decreases, reaching a maximum at infinite dilution with a Flory exponent of 0.5 . By comparing the effects of allowing and disallowing crossing in a ring melt, Brown et al. ${ }^{20}$ showed that it is the nonconcatenation constraint that makes the scaling exponent for the size of rings in melt change from 0.5 to 0.42 . Thus the effect of linear chains on the size of a ring is easily understood. In the absence of the linear chains, the nonconcatenation constraint between rings makes it difficult for rings to interpenetrate. As a result, their conformations are more compact in the melt, while in the case of ring-linear blend, linear chains can penetrate into ring coils with chain ends and the rings are therefore swollen.

Although the sample-averaged values of $R_{\mathrm{g}}^{2}$ and $A$ are nearly the same for a ring in linear matrix with different length of linear chains, the individual behavior of ring for each sample, which we denote with an overbar, is quite different. Figures 2 and 3 show, respectively, the distribution of $\overline{R_{\mathrm{g}}^{2}}$ and $\bar{A}$ from 50 samples. The data are obtained by averaging over about 20000 conformations equally spaced over the duration of the simulation runs, in essence performing a time average over each sample. It can be seen that with the increase of $N_{\text {Linear }}$, the distributions of both $\overline{R_{\mathrm{g}}^{2}}$ and $\bar{A}$ become wider. For the R30/L1000 system, the ring is quite expanded in some samples and squeezed in some other samples. The different ring sizes in the different samples reflect the differences in the state of penetration (threading) by the linear chains (see more discussion in the next paragraph). Obviously chain ends are needed for threading and the concentration of chain ends decreases with increasing length of the

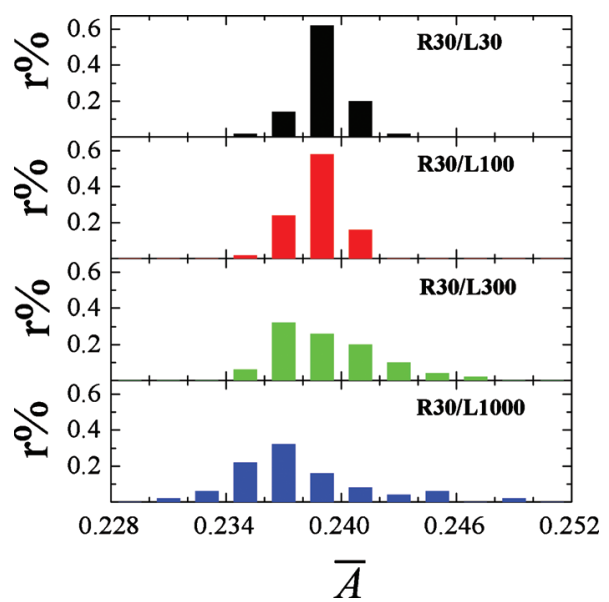

FIG. 3. Percentage distribution of the asphericity parameter from 50 samples for different chain lengths of the matrix.

linear polymers. The true equilibrium behavior for the ring conformation is the average of the different ring conformation for each threading state and of the different threading states. However, for very long linear chains, the interconversion between the different threading states becomes very slow: if a ring is not threaded by a linear chain, it is very difficult for the ring to become threaded, but once the ring becomes threaded, it takes a long time for the chain to unthread. Thus, on time scales shorter than the typical threading/unthreading time scales, the state of the ring appears nonergodic.

To visualize the different threading states, we examine the molecular conformations using the "primitive-path analysis" 43,44 with the aid of molecular dynamics. In this procedure, the polymer conformations (the positions of chain beads) are taken from MC simulations and transferred to continuous space, and both ring and linear chains are treated as beads connected by springs. The ends of linear chains and one of the ring's segments are fixed in space during the procedure. Then, the intramolecule excluded-volume interactions for linear chains and the ring are turned off, while the linear-linear and linear-ring excluded-volume interactions are retained. The energy of the system is minimized using molecular dynamics simulation by slowly cooling the system to $T=0$. Without thermal fluctuations and intrachain excludedvolume interactions, the bond springs pull the linear chains taut due to fixed chain ends and entanglements; the interchain excluded-volume interactions ensure that chains do not cut through each other. As for the ring, since one of its beads is fixed and intrachain excluded-volume interactions are turned off, all the other beads will collapse onto the fixed bead if the ring is not threaded by linear chains. If the ring is threaded, the collapse will be stopped by the excluded volume of the chain segments. We remind the readers that the size of the various entities is not the pertinent information in this analysis; it is the topological structures that are of interest. We find that threading exists widely and in some samples the ring remains threaded during the entire simulation run. Some typical snapshots are given in Fig. 4 [for a nonthreaded ring, all the beads will collapse to a point, however, for better viewing we still draw such a ring with a finite size in Fig. 


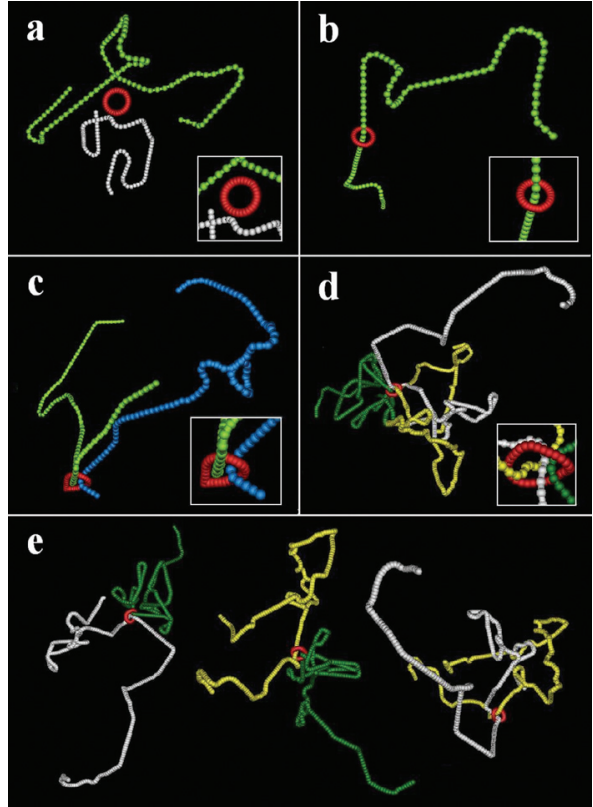

FIG. 4. Representative molecular conformations of ring-30 and matrix chains with different degrees of penetration. The original molecular coordinates are obtained from our MC simulation and then treated using a molecular dynamics method. For clarity, every three monomers of linear chains are shown as one. (a) is a schematic model; (b) and (c) are from R30/L300; (d) and (e) are from R30/L1000. (e) shows the remaining threaded states after one threading chain from (d) is removed.

4(a)], showing the once-threaded, twice-threaded, and thricethreaded states of the ring. For clarity, the different chains are shown in different colors. For comparison, the schematic molecular conformation for the unthreaded state is also given in Fig. 4. [It must be noted that the linear chains entangle heavily with each other except for $N_{\text {Linear }}=30$. However for clarity, only the linear chains that thread the ring are shown, except in the unthreaded case of Fig. 4(a).] The slow interconversion between the different threading states (to be discussed later) shown in the figure gives rise to the large dispersion in the static properties of the ring for long linear chain matrix.

We now consider the center-of-mass self-diffusion coefficient $D_{\mathrm{cm}}$ of the ring, whose classic definition is

$$
D_{\mathrm{cm}}=\lim _{t \rightarrow \infty} \frac{\left\langle\left[R_{\mathrm{cm}}(t)-R_{\mathrm{cm}}(0)\right]^{2}\right\rangle}{6 t},
$$

where $R_{\mathrm{cm}}(t)$ is the position of center-of-mass for the ring at time $t$. Note that a true constant diffusion coefficient can only be obtained for sufficiently long time when the center-ofmass motion becomes truly Brownian. However, if $t$ is not long enough to make a Brownian diffusion, we can only obtain the diffusion coefficient by extrapolation; the details are described in the Appendix. While the true self-diffusion coefficient is a single well-defined value for an equilibrium system and the average in Eq. (3) can be interpreted as either an ensemble or a time average, when the simulation time is not sufficiently long, the apparent diffusion coefficient for each sample, as obtained through an extrapolation procedure, can be different. We use the dispersion in the value of the

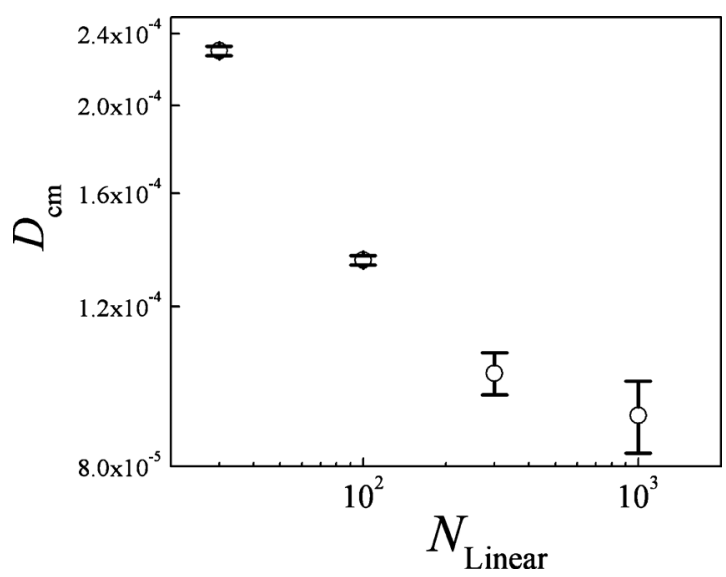

FIG. 5. The dependence of center-of-mass apparent self-diffusion coefficient of ring- 30 on the chain length of the matrix.

apparent diffusion coefficient as a measure of dynamic heterogeneity.

Mills et al. ${ }^{34}$ studied the diffusion of polymer rings in linear matrix and found the scaling exponents of $D_{\mathrm{cm}}$ on $N_{\text {Linear }}$ gradually decrease in absolute value with the increasing $N_{\text {Linear }}$, i.e., the dependence on $N_{\text {Linear }}$ becomes weaker. They explained this trend with the following consideration: for a given ring, as the chain length of the matrix increases, the CR mechanism will no longer be dominant, and the RR and R1 mechanisms become dominant. In our work, a similar trend is also found, which is shown in Fig. 5. Although the limited data do not allow us to meaningfully extract the scaling exponents of $D_{\mathrm{cm}}$ on $N_{\text {Linear }}$, the weaker dependence on $N_{\text {Linear }}$ at larger $N_{\text {Linear }}$ is consistent with the CR mechanism becoming subordinate.

Just as for the structural properties, the apparent selfdiffusion coefficient also shows large dispersion from sample to sample in the case of long linear chain matrix. In Fig. 6, we show the distribution of the apparent self-diffusion coefficients of rings in linear matrix with different chain lengths for all the 50 independent samples studied. For the R30/L30 system, relatively fast diffusion is observed as expected, with

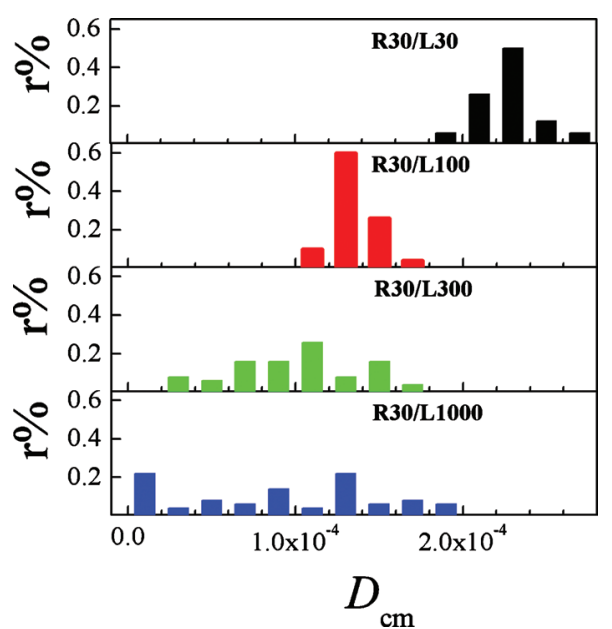

FIG. 6. Percentage distribution of the apparent self-diffusion coefficients from 50 samples for different chain length of matrix. Data for the R30/ L1000 system are taken from a simulation run of $5 \times 10^{8} \mathrm{MCS}$; all others are from the data of $1 \times 10^{8} \mathrm{MCS}$. 

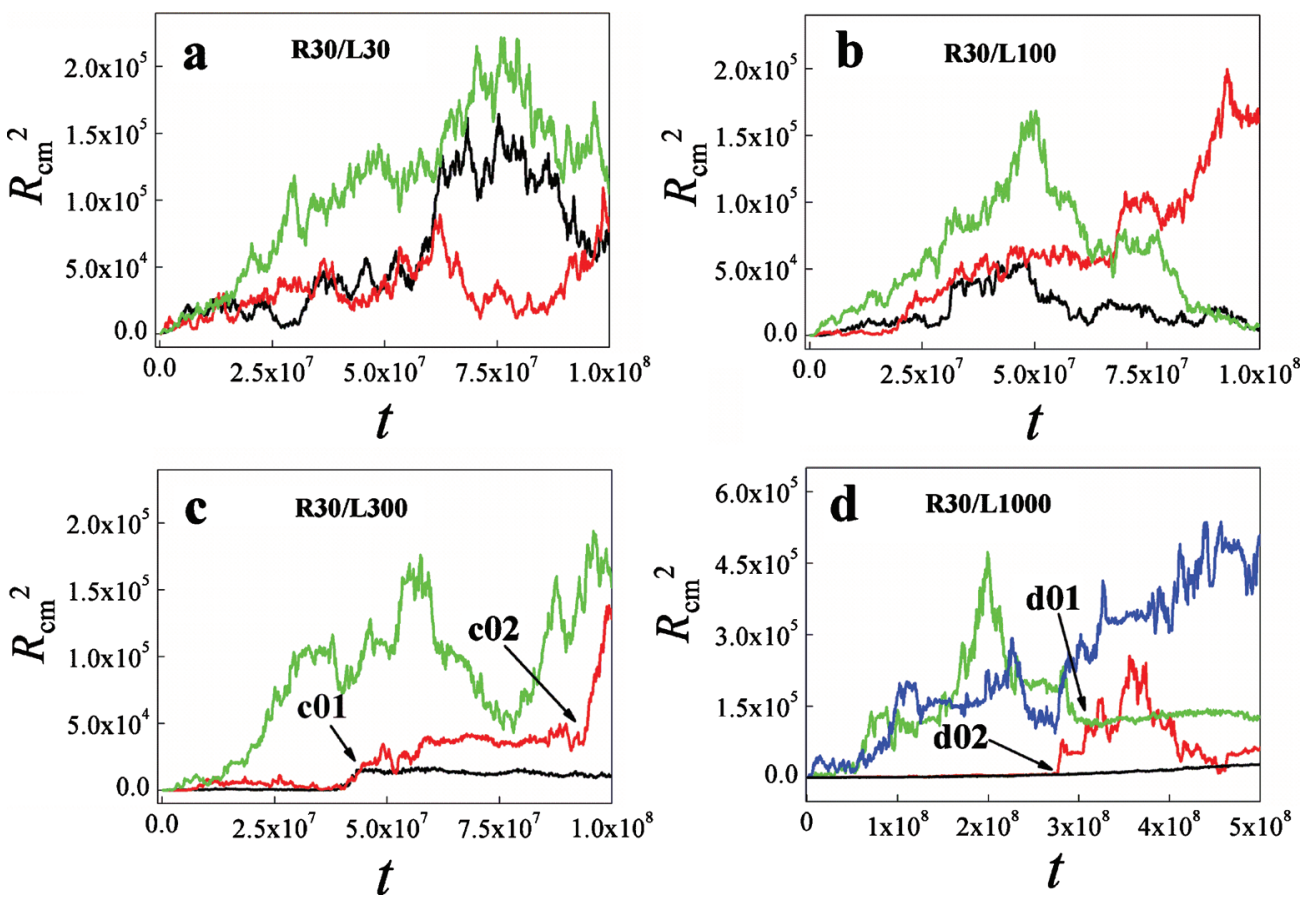

FIG. 7. Representative trajectories of ring-30 for different chain lengths of matrix. The curves from black to red, green/blue roughly correspond to decreasing degrees of threading.

a relatively narrow spread. When the length of linear chains increases to 100 , the diffusion of ring slows down significantly. With the further increase of the length of linear chains (R30/L300 system), the ring diffusion slows down further. More importantly, there seems to be a change in the ring diffusion mechanism, as indicated by the wide spread in the apparent self-diffusion coefficients. In some samples, very slow diffusion is found, which must mean significant entanglement between ring and linear chains in these samples. However in some other samples, the apparent self-diffusion coefficients are higher than those seen in the linear-30 matrix. This change in the mechanism also coincides with the apparent turn in the average diffusion coefficient with the degree of polymerization of the linear chains shown in Fig. 5. For the R30/L1000 system, the spread in the apparent diffusion coefficients become even wider, and also there appears a clustering of the samples toward a fast group and a very slow group. With a larger number of samples and better statistics, we may well see multiple peaks corresponding to the different threading states of the ring by the linear chain. However the data for these long linear matrix chains are strong evidence of dynamical heterogeneity and suggest that in these systems, the ring diffusion occurs by a combination of mechanisms.

The dynamical heterogeneity is more directly manifested by the diffusion trajectories. If a ring is not threaded by the linear chains, the diffusion will be relatively fast and behaves like the random walk. However, if the ring is threaded by the linear chains, then diffusion becomes slow, due to several hindrance mechanisms (to be discussed later). Some typical diffusion trajectories of a ring in linear chain matrix with different chain lengths are given in Fig. 7. Note that here the "diffusion trajectory" (denoted by $R_{\mathrm{cm}}^{2}$ in Fig. 7) for a certain sample is the square displacement of ring-30 center-of-mass from the initial position and zero time of the corresponding simulation sample, so its time span equals $\tau_{\text {run. }}$. For a ring diffusing in the linear matrix with short length [R30/L30, Fig. 7(a)], the random walk diffusion behavior is always found for all the samples. For the R30/L100 system [Fig. 7(b)], some samples show a short plateau [e.g., the black curve in Fig. 7(b)]. Such a plateau must be due to temporary entrapment of the ring as a result of it being threaded by linear chains. In these systems, however, the linear chains can retract or "slip" away from the ring relatively easily. With the further increase of linear chain length (to 300 and 1000 ), in most samples the diffusion trajectories exhibit extended plateau regions, indicating increased threading [Fig. $7(\mathrm{c})$ and $7(\mathrm{~d})]$. This statement is supported by primitive path analysis. For example, for the red curve in Fig. 7(c), from the corresponding molecular conformations, it is found that the ring remains threaded by chains along the segment of the trajectory between $\mathrm{c} 01$ and $\mathrm{c} 02$ (the points such as $\mathrm{c} 01$ are determined from the conformations by using the "primitivepath analysis"). After c02, the chain unthreads, and the ring begins to resume normal diffusion. For the black curve in Fig. 7(c), we also find from the analysis of the molecular conformations that at first the ring is threaded by one chain; after this chain unthreads at position $\mathrm{c} 01$, the ring is soon threaded by another chain. The plateaus become even more pronounced in Fig. 7(d). For one of the trajectories (the black one), the ring moved only a small distance during the entire simulation time, while the green trajectory first shows diffusive motion and then gets stalled at point $\mathrm{d} 01$, and the red trajectory moves a little from its initial position after a long pause and then seems to locally fluctuate after point $\mathrm{d} 02$. It is important to realize that threading alone does not necessarily stall the diffusion of the ring. However, we must bear in mind that for the long chain matrix cases, the chains them- 

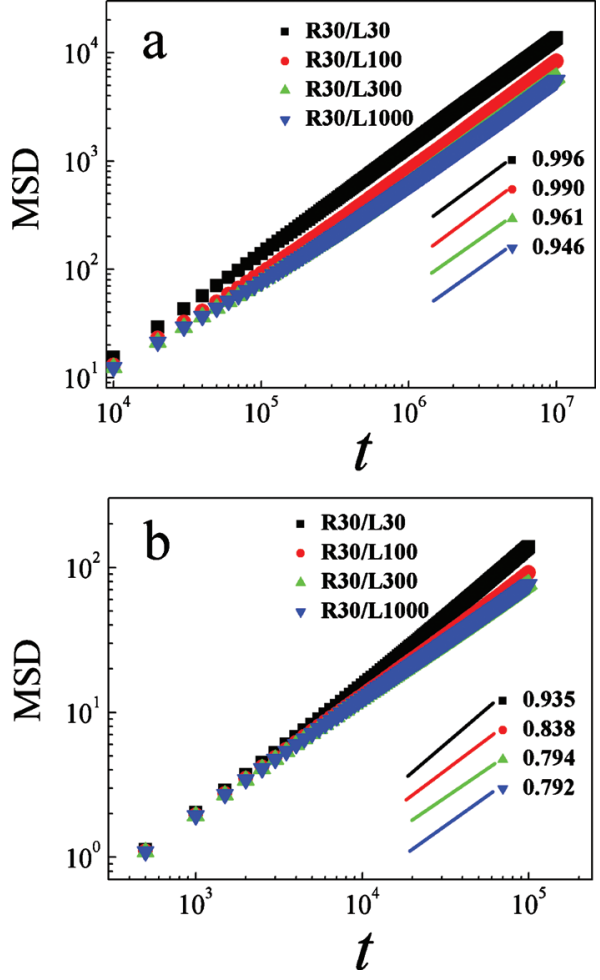

FIG. 8. Apparent scaling exponents for mean square displacement vs time (MCS) for different time spans and different chain lengths of matrix.

selves are entangled; each turn in the primitive path analysis shown in Fig. 4 corresponds to a point of entanglement. Some of these entanglements block the sliding of the ring along the threading chain, which essentially traps the ring to a segment of the chain on the order of the entanglement length.

The effect of dynamic heterogeneity is also reflected in the sample averaged center-of-mass mean-squaredisplacement (MSD) as deviation from strict linear behavior in time. As shown in Fig. 8, in order to get a better understanding about the power laws between MSD and $t$, we divide the time span into two regimes: $10^{4}$ to $10^{7}$ and $5 \times 10^{2}$ to $10^{5}$. Figure 8 (a) corresponds to the long time diffusion which is nearly Brownian, with exponents close to one; the scaling exponents (the slope of the curve in log-log figure) are $0.996,0.990,0.961$, and 0.946 for ring-30 in linear-30, 100,300 , and 1000, respectively, showing that even at these long time, there is still deviation from strict Brownian diffusion, with more deviation for the longer chain matrix. Figure 8(b) shows the behavior at shorter times, with scaling exponents $0.935,0.838,0.794$, and 0.792 , respectively, in the order of increasing matrix chain lengths; subdiffusive behavior is clear for the longer chains. Similar subdiffusive behavior has been observed for both pure linear chains ${ }^{41}$ and small rings. ${ }^{15,17}$ This non-Fickian diffusion is attributed to some net force acting on the test chain from the surrounding molecules. In our work the net force is most likely related to the surrounding linear chain matrix that constrains the ring in the form of threading, or even in "cavities" with no threading. The long life time of the constraint creates an environment that is dynamically highly correlated, leading to deviation from simple Brownian diffusion.

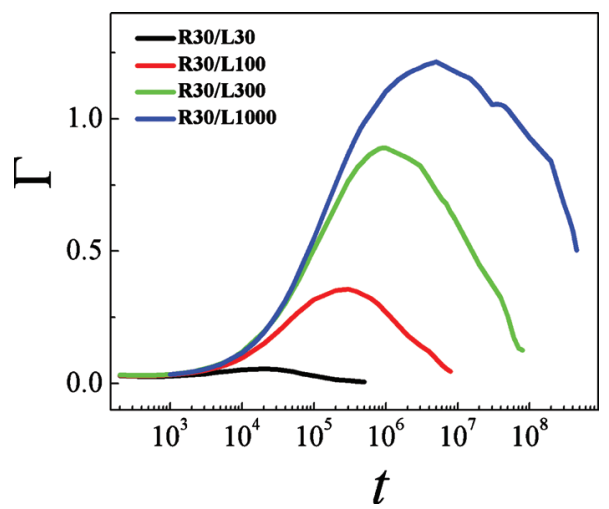

FIG. 9. The non-Gaussian parameter vs $t$ (MCS) for different chain lengths of the matrix.

A quantitative measure of the deviation from strict Brownian diffusion is the non-Gaussian parameter defined as

$$
\Gamma=\frac{3\left\langle r^{4}\right\rangle}{5\left\langle r^{2}\right\rangle^{2}}-1,
$$

where $r$ is the root MSD of center of mass. Note that $\Gamma$ should be 0 for Brownian motion obeying a Gaussian distribution, so deviation of this parameter from 0 is a measure of the degree of non-Gaussian nature. For sufficiently long time, the process must become Gaussian because of central limit theorem. From our results shown in Fig. 9, it can be seen that this long time limit is not reached by our simulation time except for the shortest chain length of 30 . For $N_{\text {Linear }}$ $=100, \Gamma$ nearly drops to zero. For the two longer chain lengths, $\Gamma$ remains significantly above 0 , although the decreasing trend at long times is clear. Another clear trend, particularly in these longer chain samples, is that the nonGaussian parameter is nonmonotonic in time-it first increases and then decreases, with the peak time increasing with increasing linear chain lengths. The height of the peak, which can be taken as a measure of the strength of the dynamic heterogeneity, also increases with increasing lengths of the linear chain. We note that non-Gaussian behavior can also be calculated by an alternative measure introduced by Flenner and Szamel ${ }^{45}$ and defined as $\Gamma=\left\langle r^{2}\right\rangle\left\langle 1 / r^{2}\right\rangle / 3-1$, which can be more effective in characterizing the nonGaussian behavior than that defined in Eq. (4). However, as this alternative definition requires the calculation of the reciprocal of the distance square, which can diverge for our lattice model at short times, we only present data using Eq. (4).

Our results and analysis given in the preceding paragraphs suggest the following physical picture for a ring diffusing in the linear chain matrix. For short chains, the high concentration of chain ends makes it quite easy for the chains to thread and unthread a ring, and the chains themselves are not entangled. So a ring is in a rapidly fluctuating, isotropic (on the length scale of the ring) environment. Its diffusion is the normal Brownian diffusion. As the length of the linear chain increases, the relaxation of the matrix itself slows down and the ring becomes increasingly entangled with the chains. When the chain length exceeds the entanglement length, the matrix forms an entangled network. Also, 
the different entangled states between the ring and the matrix chains become more distinct - a ring can be nonthreaded, threaded once, twice, or thrice-and their interconversion becomes more difficult. As a result of low concentration of chain ends, it is very difficult for a ring that is not threaded by a chain to become threaded, but once threaded, it is also difficult to unthread. In the threaded states, the diffusion of the ring is severely restricted. If the ring is threaded by multiple chains, as in the cases shown in Figs. 4(c) and 4(d), the joint action of the multiple threading chains can prevent the ring from sliding along any of the chains, thus effectively arresting the ring in a temporarily trapped state, until some of the chains unthread themselves from the ring. Note that even if some of the threading chains slither out, the constraint on the ring may still remain. For example, in Fig. 4(d), if the yellow chain is removed, the remaining white and green chains will keep the ring from moving; see the first configuration in Fig. 4(e). Therefore, for these multiply threaded ring conformations, relaxation will occur on a times scale that can be much longer than the disengagement time of the linear chains themselves. Even for the singly threaded conformation, diffusion is unlikely to take the form of a ring freely sliding along the chain contour as implied by the R1 mechanism, ${ }^{34}$ because the threading chain is entangled with other chains; these entanglement points can block the motion of the ring along the chain, leading to temporary entrapment of the ring to a segment of the chain on the order of the entanglement length. Unfortunately, with our current computational resources, we have not been able to make longer simulations to obtain a clear picture of the detailed relaxation mechanisms, or to obtain conclusive results on the duration of the heterogeneity. Also, because of the complications mentioned above, we have not been able to come up with a simple scaling estimate of the threading/unthreading time scales; such a task is left for future studies (by us or by others).

In summary, for a ring in long linear-chain matrix, our study suggests that the slow conversion between the differently threaded states gives rise to both the structural and dynamic heterogeneity on times scale that can be much longer than the relaxation time of the linear chain matrix, and the diffusion trajectory contains segments of long pauses as a result of temporary entrapments by the entangling chains.

\section{CONCLUSIONS}

In this paper, we have carried out Monte Carlo simulations on the conformation properties and the diffusion of a single ring polymer in the matrix of linear chains. The main result is that for long chain matrix, both the static and dynamic properties of the ring show strong heterogeneity, as gauged by the large spread in the distribution of these properties from different samples. Analysis of the topological state of entanglement between the ring and the linear chains using the primitive path analysis reveals that, at the same thermodynamic condition, the ring can be threaded by one chain, two chains, and multiple chains, or not threaded by any chain. In the threaded states, the diffusion of the ring is considerably slowed down or stalled in some cases, giving rise to long pauses in the diffusion trajectory, with different diffusion behavior for the differently threaded states. We argue that the life time of these differently threaded states is long-longer than the longest relaxation time of the linear chain matrix. This long conformation relaxation time is responsible for the appearance of conformational and dynamic heterogeneities on certain time scales. In other words, on these time scales, the system will appear nonergodic.

These conclusions are qualitatively consistent with the earlier suggestion ${ }^{34}$ that diffusion of rings in the linear matrix occurs by a combination of mechanisms. In a macroscopic sample, the rings will exist in several distinctly threaded states with sufficiently long life time, each state corresponding to a different diffusion mechanism. The overall average diffusion behavior is thus the weighted average of these long-lived different conformation states. However, as should become clear from our primitive path analysis, the diffusion mechanisms for the differently threaded conformation states are more complicated than the three simple mechanisms (CR, RR, and R1) mentioned in the Introduction. More important, due to the structural and dynamic heterogeneities, the ensemble averaged quantity is not a very useful informant of the system's dynamic behavior. Each conformation can be regarded as a distinct entity with its own structural and dynamic behaviors. In this sense, the differently threaded ring conformations can be considered examples of what de Gennes called "molecular individualism." tion of the dynamic behavior will have to describe the individual behavior of each of the topologically different conformers and their interconversion dynamics. Theoretically, this would call for a different strategy than the current meanfield type description of polymer dynamics in which a test chain is studied in the spatially homogeneous environment of other chains and the test chain is taken to exhibit the average behavior of other chains.

\section{ACKNOWLEDGMENTS}

This work is supported by the National Natural Science Foundation of China (NSFC) (Grant Nos. 50873098, 50930001, and 20734003) programs and the Fund for Creative Research Groups (Grant No. 50921062). L.J.A. and Z.G.W. acknowledge the financial support of NSFC (Grant No. 20620120105). Z.Y.S. acknowledges the financial support of JLSTP (Grant No. 20070113).

\section{APPENDIX: EXTRAPOLATION METHOD FOR THE APPARENT DIFFUSION COEFFICIENT}

For a given sample, the mean square center-of-mass displacement, which we denote by $\overline{R^{2}}$, is obtained by a sliding time average (using 900000 equally spaced time intervals on the trajectory as the initial positions). The apparent diffusion coefficient $D_{\mathrm{cm}}$ is obtained by plotting $\overline{R^{2}} /(6 t)$ versus $1 / t$ and extrapolating to $1 / t=0$. We find that the fitting function $y=A_{1} \exp \left(-x / b_{1}\right)+A_{2} \exp \left(-x / b_{2}\right)+y_{0}, \quad$ where $\quad x \equiv 1 / t$, $y \equiv \overline{R^{2}} /(6 t)$, and $A_{1}, A_{2}, b_{1}, b_{2}, y_{0}$ are fitting parameters, provides a good fit for all the data (with different fitting parameters for each sample). An example is shown in Fig. 10 . 


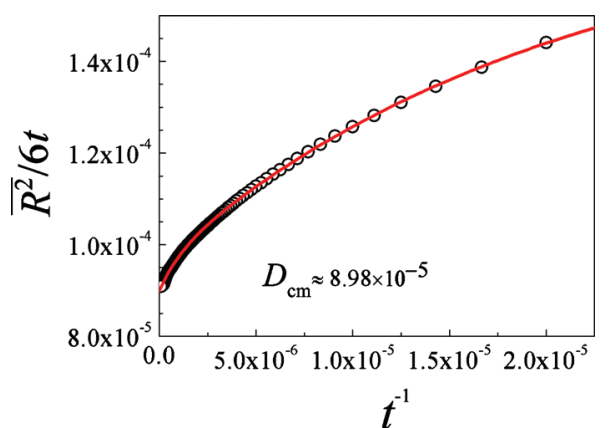

FIG. 10. Example from a R30/L1000 sample showing the extrapolation procedure to obtain the apparent self-diffusion coefficient.

${ }^{1}$ S. P. Obukhov, M. Rubinstein, and T. Duke, Phys. Rev. Lett. 73, 1263 (1994).

${ }^{2}$ T. McLeish, Science 297, 2005 (2002).

${ }^{3}$ J. Roovers, Macromolecules 18, 1359 (1985).

${ }^{4}$ G. B. McKenna, G. Hadziioannou, P. Lutz, G. Hild, C. Strazielle, C. Straupe, P. Rempp, and A. J. Kovacs, Macromolecules 20, 498 (1987).

${ }^{5}$ J. Roovers and P. M. Toporowski, J. Polym. Sci., Part B: Polym. Phys. 26, 1251 (1988).

${ }^{6}$ D. J. Orrah, J. A. Semlyen, and S. B. Ross-Murphy, Polymer 29, 1452 (1988).

${ }^{7}$ G. B. McKenna, B. J. Hostetter, N. Hadjichristidis, L. J. Fetters, and D. J. Plazek, Macromolecules 22, 1834 (1989).

${ }^{8}$ V. Arrighi, S. Gagliardi, A. C. Dagger, J. A. Semlyen, J. S. Higgins, and M. J. Shenton, Macromolecules 37, 8057 (2004).

${ }^{9}$ S. Gagliardi, V. Arrighi, R. Ferguson, A. C. Dagger, J. A. Semlyen, and J. S. Higgins, J. Chem. Phys. 122, 064904 (2005).

${ }^{10}$ A. Takano, Polym. Prepr. Jpn. 56, 2424 (2007).

${ }^{11}$ M. E. Cates and J. M. Deutsch, J. Phys. (Paris) 47, 2121 (1986).

${ }^{12}$ M. Rubinstein, Phys. Rev. Lett. 57, 3023 (1986).

${ }^{13}$ G. E. Garas and M. K. Kosmas, J. Chem. Phys. 108, 376 (1998).

${ }^{14}$ B. V. S. Iyer, A. K. Lele, and V. A. Juvekar, Phys. Rev. E 74, 021805 (2006).

${ }^{15}$ M. Müller, J. P. Wittmer, and M. E. Cates, Phys. Rev. E 53, 5063 (1996).

${ }^{16}$ S. Brown and G. Szamel, J. Chem. Phys. 108, 4705 (1998).

${ }^{17}$ S. Brown and G. Szamel, J. Chem. Phys. 109, 6184 (1998).
${ }^{18}$ J. M. Deutsch, Phys. Rev. E 59, R2539 (1999).

${ }^{19}$ M. Müller, J. P. Wittmer, and M. E. Cates, Phys. Rev. E 61, 4078 (2000).

${ }^{20}$ S. Brown, T. Lenczycki, and G. Szamel, Phys. Rev. E 63, 052801 (2001).

${ }^{21}$ R. Ozisik, E. D. von Meerwall, and W. L. Mattice, Polymer 43, 629 (2002).

${ }^{22}$ K. Hur, R. G. Winkler, and D. Y. Yoon, Macromolecules 39, 3975 (2006).

${ }^{23}$ J. Suzuki, A. Takano, and Y. Matsushita, J. Chem. Phys. 129, 034903 (2008).

${ }^{24}$ M. Kapnistos, M. Lang, D. Vlassopoulos, W. Pyckhout-Hintzen, D. Richter, D. Cho, T. Chang, and M. Rubinstein, Nature Mater. 7, 997 (2008).

${ }^{25}$ R. M. Robertson and D. E. Smith, Proc. Natl. Acad. Sci. U.S.A. 104, 4824 (2007).

${ }^{26}$ R. M. Robertson, S. Laib, and D. E. Smith, Proc. Natl. Acad. Sci. U.S.A. 103, 7310 (2006)

${ }^{27}$ R. M. Robertson and D. E. Smith, Macromolecules 40, 3373 (2007).

${ }^{28}$ B. V. S. Iyer, A. K. Lele, and S. Shanbhag, Macromolecules 40, 5995 (2007).

${ }^{29}$ G. Subramanian and S. Shanbhag, Macromolecules 41, 7239 (2008).

${ }^{30}$ G. Subramanian and S. Shanbhag, Phys. Rev. E 77, 011801 (2008).

${ }^{31}$ S. Nam, J. Leisen, V. Breedveld, and H. W. Beckham, Macromolecules 42, 3121 (2009).

${ }^{32}$ W. W. Graessley, Adv. Polym. Sci. 47, 67 (1982)

${ }^{33}$ J. Klein, Macromolecules 19, 105 (1986).

${ }^{34}$ P. J. Mills, J. W. Mayer, E. J. Kramer, G. Hadziioannou, P. Lutz, C. Strazielle, P. Rempp, and A. J. Kovacs, Macromolecules 20, 513 (1987).

${ }^{35}$ T. T. Perkins, D. E. Smith, and S. Chu, Science 276, 2016 (1997).

${ }^{36}$ P. G. de Gennes, Science 276, 1999 (1997).

${ }^{37}$ J. S. Shaffer, J. Chem. Phys. 101, 4205 (1994).

${ }^{38}$ J. S. Shaffer, J. Chem. Phys. 103, 761 (1995).

${ }^{39}$ M. Doi and S. F. Edwards, The Theory of Polymer Dynamics (Oxford University, New York, 1986).

${ }^{40}$ S. Shanbhag and R. G. Larson, Phys. Rev. Lett. 94, 076001 (2005).

${ }^{41}$ W. Paul, K. Binder, D. W. Heermann, and K. Kremer, J. Chem. Phys. 95, 7726 (1991).

${ }^{42}$ M. Bishop and C. J. Saltiel, J. Chem. Phys. 88, 3976 (1988).

${ }^{43}$ R. Everaers, S. K. Sukumaran, G. S. Grest, C. Svaneborg, A. Sivasubramanian, and K. Kremer, Science 303, 823 (2004).

${ }^{44}$ S. K. Sukumaran, G. S. Grest, K. Kremer, and R. Everaers, J. Polym. Sci., Part B: Polym. Phys. 43, 917 (2005).

${ }^{45}$ E. Flenner and G. Szamel, Phys. Rev. E 72, 011205 (2005). 